\title{
Laboratory Orientation Programme for Interns, A Need Based Training to Enhance Patient Care
}

\author{
Kumaran, S, Ponniraivan, K., Velayutharaj, T., Sundhararajan, N.L.
}

\begin{abstract}
Laboratory errors are classified as pre-analytical, analytical and post-analytical errors. It has been well documented that pre-analytical errors contribute to the majority of laboratory errors. Pre-analytical errors can occur in association with any process during sample collection and processing. It was found that pre-analytical errors occur at the hands of interns owing to their lack of adequate knowledge on pre-analytical procedures and processing. Adequate training of interns to provide basic scientific knowledge on laboratory issues and technical skills will enhance the quality of reports issued. The present study was conducted at Chennai Medical College and Research Centre, Tiruchirappalli, Tamilnadu, India. Thirty six newly recruited interns were included in the study. Standard questionnaires were developed to evaluate technical skills; a pre-test was conducted to assess basic knowledge on laboratory issues. The laboratory orientation programme conducted by faculty of the clinical biochemistry department included lectures on sample collection, pre-analytical errors, reference ranges and critical alert values. This was followed by a post test, conducted to assess the impact of training. There was a significant $(p<.001)$ improvement in the performance of interns in the post test (mean score: $17.61 \pm 2.3$ ) when compared with the pre-test (mean: $10.75 \pm 1.4$ ). This shows that there was a lacuna among the interns regarding the basics of laboratory methods before the training and the training programme has improved their knowledge in laboratory medicine. The laboratory orientation programme for all interns involved in the pre-analytical phase will strengthen their knowledge and skills which will enhance issuing of quality, error free reports.
\end{abstract}

\section{Introduction}

Laboratory reports serve as the hub in today's modern medicine. In other words, laboratory reports serve as a key factor in diagnostic, prognostic and therapeutic aspects of the disease. The laboratory discipline is witnessing spectacular improvement, however at the same time laboratory errors occur too. Laboratory errors lead to unnecessary stress to the patients, physicians, loss of reputation of the laboratory and also of the hospital management (Kalra, 2004).

Laboratory errors are classified as preanalytical, analytical and post analytical errors (McSwiney \& Woodrow, 1969).

Department of Biochemistry,

Chennai Medical College Hospital \& Research Centre (SRM Group),

Irungalur, Tiruchirappalli, Tamil Nadu, India.

Corresponding Author:

Senthil Kumaran

Department of Biochemistry,

Chennai Medical College Hospital \& Research Centre (SRM Group),

Irungalur, Tiruchirappalli, Tamil Nadu, India.

E Mail: sansenpran@rediffmail.com
It has been well documented that the preanalytical phase contributes much to laboratory errors (Binita et al., 2010). Pre-analytical errors can occur in various forms, starting from inadequate instructions given to patients, compliance by patients, sample collection, sample processing and transport by phlebotomists, staff nurses, technicians, duty doctors (Giuseppe et al., 2006; Young, 2003). There could be many reasons for pre-analytical errors; errors due to incorrect sample collection, incorrect sample preservation, incorrect sample request, illegible sample request, misinterpreting the test, sample interchange, haemolysis of the sample due to improper collection and processing and also patient related errors due to non-compliance to the instructions given by phlebotomists. Laboratory errors in the pre-analytical phase occur due to suboptimal knowledge of interns about sample collection, processing and transport. Proper baseline knowledge about laboratory functioning is quintessential for all interns.

The present study aims at assessing basic scientific knowledge on laboratory issues, technical skills, communication skills among all interns. Based on the assessment, a laboratory orientation programme is conducted for all interns involved in the pre-analytical phase to 
strengthen their knowledge and skills which will enhance issuing of quality reports.

\section{Materials and Methods}

The present study was conducted at Chennai Medical College and Research Centre, Tiruchirappalli, Tamilnadu, India. Thirty six newly recruited interns were included in the present study. A standard questionnaire was developed to evaluate technical skills and basic knowledge on laboratory issues, of all interns involved in the laboratory pre-analytical phase, to assess their baseline knowledge. The maximum score for the questionnaire (both preand post-test) was 20 marks. The areas enlisted in the standard questionnaire were assessment of the technique of sample collection, basic scientific knowledge on ideal samples for testing, use of proper anticoagulants, sample request completion, sample handling, sample transport to central laboratory, instructions to be given to the patients for collecting fasting samples and ensuring the adherence of patients to the instructions. Based on the pre-test marks, the laboratory orientation programme was conducted by faculty members in the Clinical Biochemistry department. The impact of training was assessed by conducting a post test, as a measure of outcome of the laboratory orientation programme.

\section{Results}

The total score for both the pre and post-test was 20 marks. Results are shown in Figure 1. The mean mark scored for the pre-test was

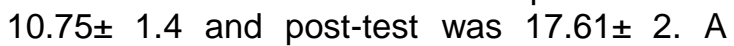
significant $\quad(p<0.001) \quad$ improvement was observed in the performance of the interns in the post test (mean score-17.61 \pm 2.3 ) when compared with the pre-test (mean-10.75 \pm 1.4 ). This shows that there was a lacuna among the interns regarding the basics of laboratory methods initially and the training programme has improved their knowledge in laboratory medicine.

Figure 1: Scores of interns in pre-test and post-test.

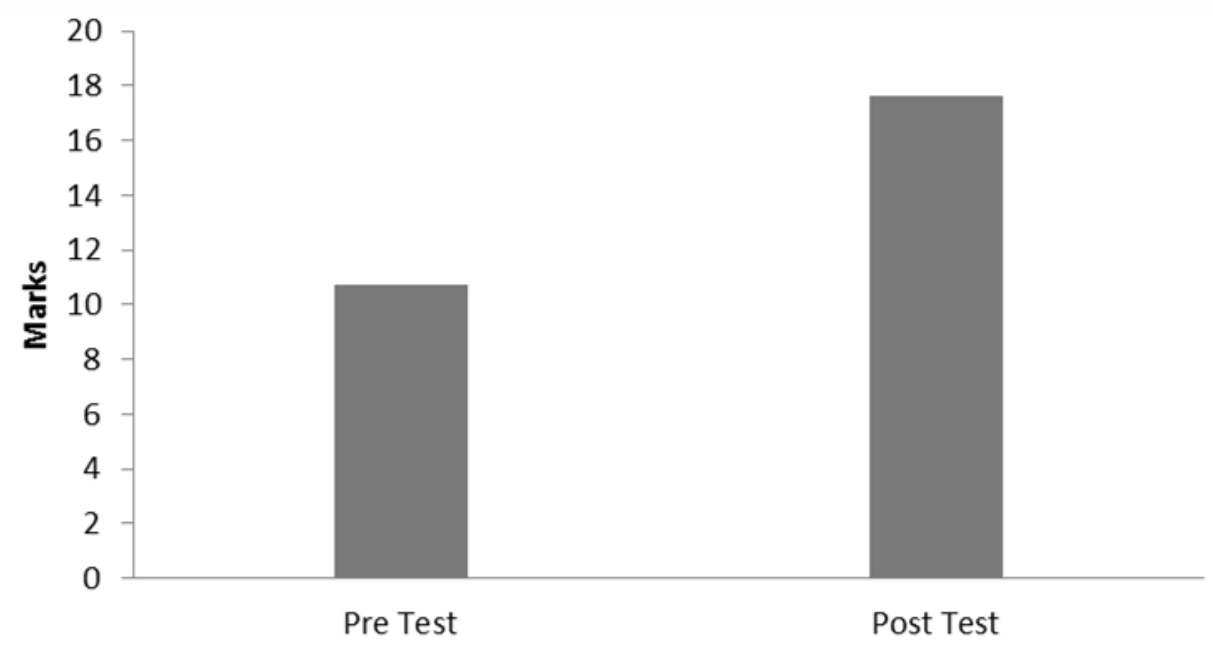

\section{Discussion}

Laboratory reports play a pivotal role in today's practice. Error free reports and timely results will enhance patient care. The laboratory work flow is divided into three main phases, preanalytical, analytical and post analytical. Preanalytical phase extends from instructing the patients for pre-analytical preparation, sample collection and sample transport to the laboratory. Analytical phase starts from receiving the sample and analyzing the sample in the vicinity of the laboratory itself. Post analytical phase is getting the results and typing and delivering the appropriate results. In spite of great scientific advancement in the laboratory field, errors do occur. Since the preanalytical phase is handled by many personnel such as interns, ward staff, duty doctors and patients there is a high frequency of laboratory errors occurring in this stage (50-55\%), while the remaining is shared by analytical and post analytical errors. The sensitization about correct sample request form completion with necessary information about the patient such as age, sex, date and time of sample collection and clinical diagnosis documented legibly is also relevant in correlation and interpretation of the laboratory reports with the patient's clinical status. 
Bar-coding the sample is the scientific way to check the uniqueness and integrity of the sample. It was documented in a previous study that errors in the pre-analytical phase occur during the first month of internship postings (Chmura, 1987). This is attributed to lack of baseline knowledge, adequate training and awareness of laboratory errors by the interns. Interns entering into clinical postings will have suboptimal knowledge about sample collection, reference intervals, and interpretation of reports. Training and introducing newly posted interns to basic laboratory knowledge will be one way of keeping a check in limiting the pre-analytical errors in a medical college setup.

With this background the present study assessed the baseline knowledge about every sphere of the pre-analytical phase such as sample collection, use of proper anticoagulants, sample request completion, sample handling, sample transport to central laboratory, instructions to be given to the patients for collecting fasting samples and ensuring the adherence of patients to the instructions. Baseline knowledge was assessed by a standard questionnaire with a maximum score of 20 marks. The laboratory orientation programme was conducted for all interns by faculty of the Clinical Biochemistry department, Chennai Medical College Hospital and Research Centre, Tamilnadu, India. The training emphasized various aspects of the pre-analytical process such as correct sample request form completion without any ambiguity, sample collection and processing, reference range and interpretations.

The impact of training was assessed after the training as a measure of outcome of the programme. There was significant $(p<.001)$ improvement in the performance of the interns in the post test (mean score-17.61 \pm 2.3 ) when compared with pre-test (mean score -10.75 \pm 1.4). This shows that there is lack of adequate baseline knowledge about clinical biochemistry and by imparting basic scientific knowledge and providing training about the pre-analytical issues in sample collection, there is an improvement in the various aspects of clinical biochemistry as evidenced in the post test. This strategy will improve not only the scientific knowledge and skill of the interns but also help in reducing pre-analytical issues. The limitation of the present study is that the quality indicators such as sample rework, sample errors were not analyzed. Further studies are needed to evaluate the effectiveness of the training by analyzing the quality indicators such as sample rejection, sample rework.

\section{Conclusion}

Pre-analytical errors have their own consequences in diagnostic, prognostic and therapeutic aspects. The frequency of preanalytical error is more when compared to analytical and post analytical errors. Training, awareness and sensitization regarding laboratory issues will help in reducing preanalytical errors. It was found that interns lack the basic knowledge of clinical biochemistry and laboratory methods regarding sample collection, preservation and processing. A laboratory orientation programme for all interns will strengthen their knowledge and skills in clinical biochemistry and laboratory procedures, which will enhance issuing of quality, error free reports.

\section{References}

Binita, G., Bhawna, S., Ranjna, C. \& Venkatesan, M. (2010) Evaluation of Errors in a clinical Laboratory: a One - year experience, Clinical Chemistry Lab Medicine, 48, 1, pp. 63-66.

Bonini, A., Ceriotti, F., Plebani, M. \& Rubboli, F. (2002) Errors in laboratory medicine, Clinical Chemistry, 48, pp. 691-698.

Chmura, A. (1987) Measurement of physician specimen-handling errors and its contribution to laboratory information system quality, Journal Medical Systems, 11, 2, pp.95-103.

Giuseppe, L., Gian Cesare, G., Camilla, M. \& Mario, P. (2006) Pre-analytical variability: The dark side of the moon in laboratory testing, Clinical Chemistry Lab Medicine, 44, 4, pp. 358-365.

Kalra, J. (2004) Medical errors: Impact on clinical laboratories and other critical areas, Clinical Biochemistry, 37, pp. 1052-1062.

McSwiney, R. \& Woodrow, A. (1969) Types of error within a clinical laboratory, Journal of Medical Lab Technology, 26, pp. 340-346.

Young, D. (2003) Conveying the importance of the preanalytical phase, Clinical Chemistry Lab Medicine, 41, pp. 884-887. 